\title{
Automated hiPSC culture and sample preparation for 3D live cell microscopy
}

Mackenzie E. Coston ${ }^{1}$, Benjamin W. Gregor ${ }^{1}$, Joy Arakaki, Antoine Borensztejn, Thao P. Do, Margaret A. Fuqua, Amanda Haupt, Melissa C. Hendershott, Winnie Leung, Irina A. Mueller, Angelique M. Nelson, Susanne M. Rafelski, Madison J. Swain-Bowden, W. Joyce Tang, Derek J. Thirstrup, Winfried Wiegraebe, Calysta Yan, Ruwanthi N. Gunawardane, Nathalie Gaudreault $^{2}$

Affiliations: Allen Institute for Cell Science, 615 Westlake Ave N, Seattle, WA, 98125, USA.

${ }^{1}$ These authors contributed equally

${ }^{2}$ Corresponding Author

Contact Information: nathalieg@alleninstitute.org

\section{Summary}

Our goal is to identify and understand cellular behaviors using 3D live imaging of cell organization. To do this, we image human inducible pluripotent stem cell (hiPSC) lines expressing fluorescently tagged protein representing specific cellular organelles and structures. To produce large numbers of standardized cell images, we developed an automated hiPSC culture procedure, to maintain, passage and Matrigel coat 6-well plastic plates and 96-well glass plates compatible with high-resolution 3D microscopy. Here we describe this system including optimization procedures and specific values for plate movement, angle of tips, speed of aspiration and dispense, seeding strategies and timing of every step. We validated this approach through a sideby-side comparison of quality control results obtained from manual and automated methods. Additionally, we developed an automated image-based colony segmentation and feature extraction pipeline to predict cell count and select wells with consistent morphology for high resolution 3D microscopy.

\section{Introduction}

Images of cells contain rich information about their shape and cellular structure organization that can be used to predict and understand cellular behaviors in both normal and pathological conditions. We aim to develop these models by combining single cell genomics, transcriptomics, 3D imaging, and phenotypic data. To train these models, we needed large, high quality image datasets and produced them using a robust, high throughput imaging pipeline that includes automated cell culturing and preparation of optical grade glass plates for 3D high resolution imaging (Figure 1A).

We used human induced pluripotent stem cells (hiPSCs) because of their many advantages. hiPSCs provide a diploid, non-transformed cellular context that can be propagated indefinitely and give rise to many differentiated cell types. They also show enormous promise for personalized medicine as "disease in a dish" models as well as for regenerative medicine (Mahla, 2016). Their relative karyotypic stability over dozens of passages in culture affords high reproducibility Additionally, the cells can also be genome edited to produce fluorescently tagged 
Automated hiPS cell culture

Allen Institute for Cell Science

proteins that can be readily visualized. The Allen Cell Collection (https://www.allencell.org) is a set of gene-edited cell lines, harboring a fluorescently tagged protein that localizes to a cellular structure (Roberts et al., 2017). They are available from Coriell (https://www.coriell.org/1/AllenCellCollection) and used for this study.

Here we provide an automated cell culture platform designed to grow and expand geneedited hiPSC lines on traditional 6-well plastic dishes as well as imaging samples of hiPSCs seeded on optical grade glass bottom 96-well plates. The automated cell culture platform is based on robotic equipment and image processing algorithms that serve to produce consistent cell morphology, as well as ensuring reproducibility, standardization, and scalability. The automated cell culture methodology was developed by replicating and automating key details of a manual cell culture method (Roberts et al., 2017). Important details include the speed of media aspiration and dispensing, the speed of plate movement on the deck, the relative position and angle of tips into wells, the temperature of culture and solution, and the time of incubation. A side-by-side assessment of the morphology, growth rate, stemness markers expression and cytogenetic analysis of our hiPSC lines cultured on the automated platform or handled manually by experts, allowed us to modify and optimize each step and key parameter until achieving comparable culture quality. To our knowledge, this is the first successful report on the automated seeding of hiPSCs on 96-well optical grade glass bottom plates enabling high quality, live cell 3D microscopy of stem cells at a large scale. Our intention is for any research labs interested in working with hiPSCs to easily reproduce some or all parts of this platform using their own equipment. 


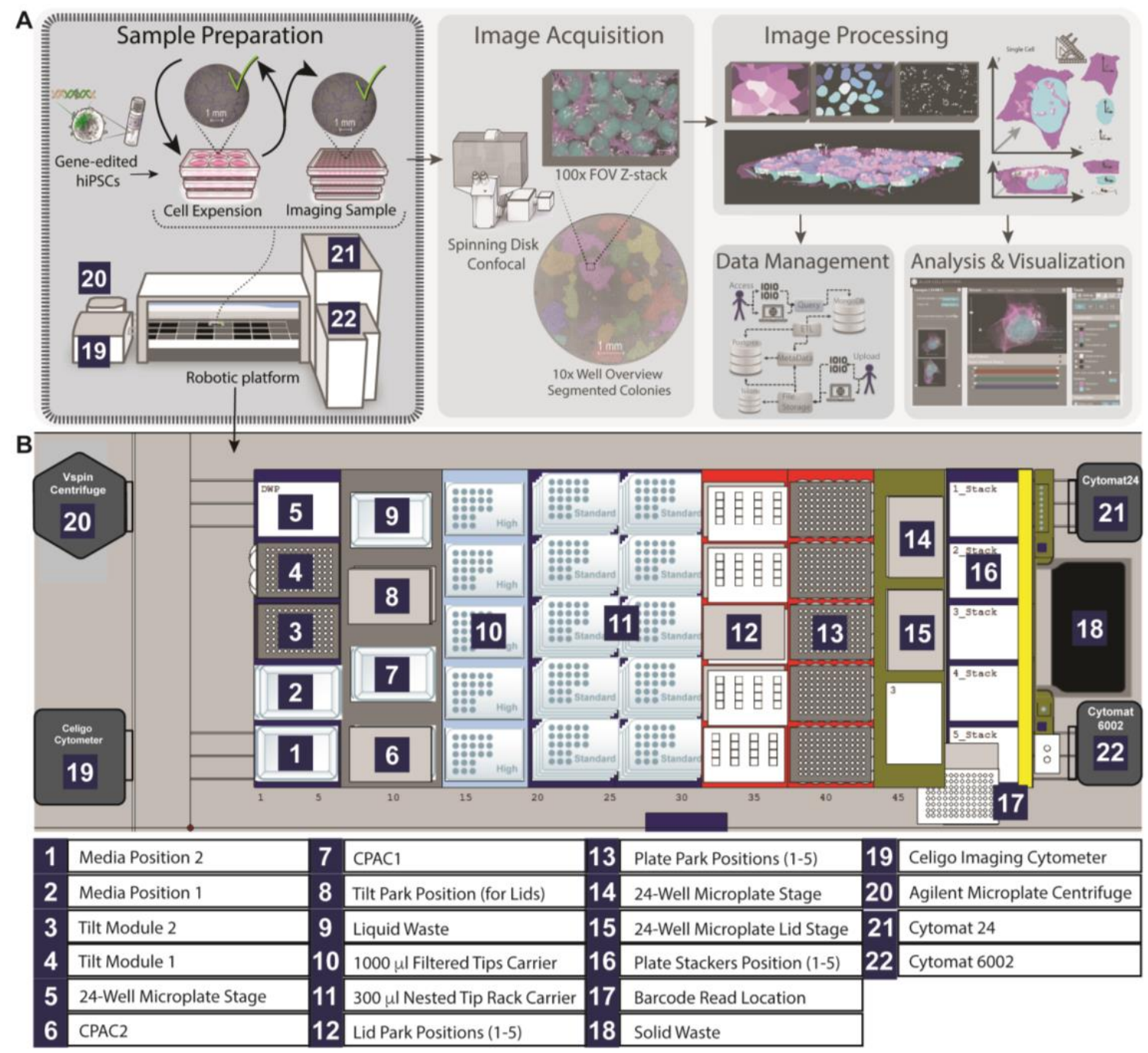

Figure 1: Allen Institute imaging pipeline workflow: (A) The imaging pipeline used to generate the Allen Cell Image collection starts from a frozen vial of gene-edited hiPSCs. The cells are expanded, passaged, and then seeded on glass plates and imaged live on a spinning-disk confocal microscope. Cells are segmented, and quantitative features are extracted from the images for analysis and modeling. In this manuscript we describe the automation and standardization of the sample preparation which includes workflows for cell expansion and seeding on glass plates. (B) Robotic platform layout: the general deck layout of the Hamilton Robotics Microlab STAR Liquid Handling System with individual components labeled 1-18. Additional integrated components in series are labeled 19-22. Scale bar is $10 \mu \mathrm{m}$ unless otherwise stated.

\section{Results}

\section{Automated Cell Culture Method Development Daily cell culture workflow}

Adaptations to the original manual cell culture method (Roberts et al., 2017) are outlined in Table 1. They fell into three distinct categories: our robotic equipment, method optimization, and practicality and consistency. For example, we used different cell culture plates and modified how plates and reagents were warmed or cooled on the robotic platform and optimized other aspects of the method by replacing qualitative assessments of the cell morphology and growth 


\section{Automated hiPS cell culture \\ Allen Institute for Cell Science}

with quantitative measurements using image processing approaches. Overall, $75 \%$ of the changes made to the original manual method were designed to adapt to the robotic equipment limitations.

\section{Table 1. Adaptations and differences between manual and automated methods}

\begin{tabular}{|c|c|c|c|}
\hline Workflow & Key Steps & Manual Method & Automated Method \\
\hline \multirow{2}{*}{ 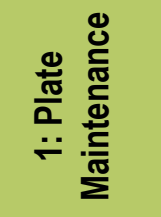 } & Media preparation & mTeSR1 stock stored in filtered bottles & mTeSR1 stored in lidded reservoirs \\
\hline & $\begin{array}{l}\text { Media RT } \\
\text { equilibration }\end{array}$ & Allow mTeSR 1 to come to RT for $30 \mathrm{~min}$ to $1 \mathrm{hr}$ & $\begin{array}{l}\text { mTeSR1 reservoir placed onto CPAC, } \\
\text { at } 34^{\circ} \mathrm{C} \text { for } 30 \mathrm{~min}\end{array}$ \\
\hline \multirow{12}{*}{ 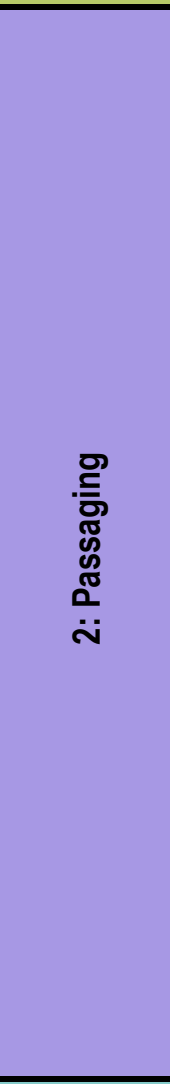 } & $\begin{array}{r}\text { Accutase } \\
\text { warming }\end{array}$ & Water bath at $37^{\circ} \mathrm{C}$ or RT & $\mathrm{CPAC}$ at $37^{\circ} \mathrm{C}$ \\
\hline & Cell collection & $15 \mathrm{~mL}$ centrifugal tubes & 24-well lidded microplate \\
\hline & \# of cell lines & Max two cells lines using $10 \mathrm{~cm}$ source plate & Up to six cell lines using 6-well plate (Figure $3 \mathrm{H}$ ) ${ }^{*}$ \\
\hline & Quality control & $\begin{array}{c}\text { Qualitative assessment: } \\
\text { Manual inspection under microscope } \\
\end{array}$ & \begin{tabular}{|c|}
\multicolumn{1}{|c|}{ Quantitative assessment: } \\
Image acquisition \& processing/algorithm classification (Figure 4) **
\end{tabular} \\
\hline & $\begin{array}{l}\text { Media and PBS } \\
\text { wash }\end{array}$ & $\begin{array}{l}\text { Hand tilting of plate at approximate angle/hand- } \\
\text { controlled aspiration and dispense from side of well }\end{array}$ & $\begin{array}{l}\text { Plate tilted by tilt module at consistent angle, } \\
\text { aspiration, and dispense speed from side of well (Table S3) }\end{array}$ \\
\hline & Trituration & $\begin{array}{l}\text { Trituration at subjective speed with } 1 \mathrm{~mL} \text { pipette, } \\
\text { rinsing the cells across the vessel surface } 8-10 \text { times }\end{array}$ & $\begin{array}{l}\text { Plate tilted and two } 1,000 \mu \mathrm{L} \text { channels/well aspirate from the } \\
\text { lowest point and dispense at four locations in the well at a precise, } \\
\text { constant speed (Figure 3A, Table S3) }\end{array}$ \\
\hline & Centrifugation & $\begin{array}{c}15 \mathrm{~mL} \text { centrifugal tubes placed in a Thermo Fischer } \\
\text { Compact Ventilated Centrifuge }\end{array}$ & Robot-accessible Agilent Centrifuge with 24-well microplate \\
\hline & $\begin{array}{l}\text { Aspiration of } \\
\text { supernatant }\end{array}$ & $\begin{array}{l}\text { Aspirate off the supernatant on opposite side of pellet } \\
\text { by tilting } 15 \mathrm{~mL} \text { tube one at a time }\end{array}$ & $\begin{array}{l}\text { Aspiration location of tips in wells (Figure } 3 \mathbf{G} \text { ) is predetermined } \\
\text { based on consistent pellet position and performed simultaneously }\end{array}$ \\
\hline & $\begin{array}{l}\text { Resuspension of } \\
\text { cell pellet }\end{array}$ & $\begin{array}{l}\begin{array}{c}\text { Resuspend pellet with handheld pipette until cell pellet } \\
\text { is fully dispersed }\end{array}\end{array}$ & $\begin{array}{c}\text { All aspiration and dispense steps are at consistent speed and } \\
\text { distance from the bottom of the 24-well plate and performed with } 1 \\
\mathrm{~mL} \text { tips and two mixing cycles (Figure } 3 \mathrm{G} \text {, Table S3) }\end{array}$ \\
\hline & $\begin{array}{l}\text { Cell seeding } \\
\text { preparation }\end{array}$ & $\begin{array}{l}\text { Cells seeded straight from cell suspension following } \\
\text { gentle mixing }\end{array}$ & $\begin{array}{c}\text { Intermediate step: cell suspension mixed and diluted in row B-C of } \\
\text { 24-well microplate to create [final seeding] of the 6- and 96-well } \\
\text { plates (Table S3) }\end{array}$ \\
\hline & Cell seeding & $\begin{array}{c}\text { Dispense cell solution dropwise to cell culture plate } \\
\text { containing mTeSR1+ ROCK Inhibitor, location of } \\
\text { seeding, volume of suspension and dispense speed } \\
\text { varies }\end{array}$ & $\begin{array}{l}\text { 96-well plate: cell suspension aspirated after two mixes and } \\
\text { dispensed along the side of the well (Figure 3D) } \\
\text { 6-well plate: cell suspension dispensed in five locations in each } \\
\text { well (Figure 3B) }\end{array}$ \\
\hline & Cell distribution & $\begin{array}{l}\text { Slide tissue culture plates front-to-back and side-to- } \\
\text { side to ensure even cell distribution at least 2-4 times } \\
\text { and keep level for even cell attachment }\end{array}$ & $\begin{array}{l}\text { Tips dispense cell suspension above defined locations at defined, } \\
\text { consistent speed (Figure 3B\&D; Table S3) } \\
\text { 6-well plate is moved swiftly across the deck to multiple points to } \\
\text { further distribute cell }\end{array}$ \\
\hline \multirow{6}{*}{ 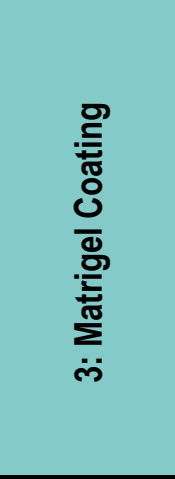 } & Storage & Two steps: $-80^{\circ} \mathrm{C} \mathrm{C}(24 \mathrm{hr})$ then $-20^{\circ} \mathrm{C}(<2$ weeks $)$ & One step: $-20 \mathrm{C}(<3$ months $)$ ** \\
\hline & Aliquots & $\begin{array}{c}1 \mathrm{~mL} \text { aliquots dispensed with serological pipettes pre- } \\
\text { chilled at }-20^{\circ} \mathrm{C} \text { into } 50 \mathrm{~mL} \text { centrifuge tubes; tubes } \\
\text { stored at }-20^{\circ} \mathrm{C}\end{array}$ & $1 \mathrm{~mL}$ aliquots into one side of a 2 -column reservoir; stored at $-20^{\circ} \mathrm{C}$ \\
\hline & [Working] & $0.337 \mathrm{mg} / \mathrm{mL}$ & $0.185 \mathrm{mg} / \mathrm{mL}^{*}$ \\
\hline & Mixing & $\begin{array}{c}\text { Pre-chilled 25-50 mL pipette and triturate 3-5 times on } \\
\text { ice }\end{array}$ & $\begin{array}{l}\text { CPAC cooled to } 4^{\circ} \mathrm{C} \text {, multiple } 1 \mathrm{~mL} \text { pipette tips and two mixing } \\
\text { cycles using fast aspiration and dispense speeds (Table S3) }\end{array}$ \\
\hline & Coating & \begin{tabular}{|c|}
$\begin{array}{c}\text { Dispense into center of cell culture plate trying to avoid } \\
\text { bubbles or splash-back; rotate plate evenly to coat } \\
\text { entire surface }\end{array}$ \\
\end{tabular} & $\begin{array}{l}\text { Defined and consistent dispense speeds (Table S3) and locations } \\
\text { (Figure 3C\&E) within wells to coat entire surface }\end{array}$ \\
\hline & $\begin{array}{l}\text { Coated plate } \\
\text { storage }\end{array}$ & \begin{tabular}{|c|} 
Same day use after incubation at RT for $1 \mathrm{hr}$ or be \\
stored for up to two weeks at $4^{\circ} \mathrm{C}$; incubate for $15 \mathrm{~min}$ \\
at RT, if stored at $4^{\circ} \mathrm{C}$ for longer than $1 \mathrm{hr}$ before \\
Matrigel is removal
\end{tabular} & $\begin{array}{l}\text { Stored at } 4^{\circ} \mathrm{C} \text { overnight to one week; } \\
\text { incubated at } 37^{\circ} \mathrm{C} \text { for } 2 \text { hrs before Matrigel removal }\end{array}$ \\
\hline
\end{tabular}

Most adaptations were implemented due to robotic equipment constraints, but some adaptions were implemented for method optimization* or practicality and consistency**. 
Automated hiPS cell culture

Allen Institute for Cell Science

The automated cell culture platform can be used for both cell expansion and preparation of plates for 3D live cell imaging (Figure 1A). The cell expansion starts with a frozen vial of cells which are grown on Growth Factor Reduced (GFR) Matrigel pre-coated, plastic 6-well plates for up to ten passages. This is followed by stringent quality controls (QCs) to ensure the cells maintained characteristic stemness morphology, marker expression, and stable cytogenetic throughout the data collection period. At each passage, new sample preparation workflow begins with Matrigel coating of the glass bottom 96 -well plates, followed by cell seeding the subsequent day in the center 60 wells of the 96 -well plates, leaving the outer rows and columns empty. Media is changed daily for both 6 -well and 96 -well plates. All plates are also imaged at low magnification, 4 days post seeding, and overview images are processed to predict cell count and select best morphology for high quality and resolution 3D imaging.

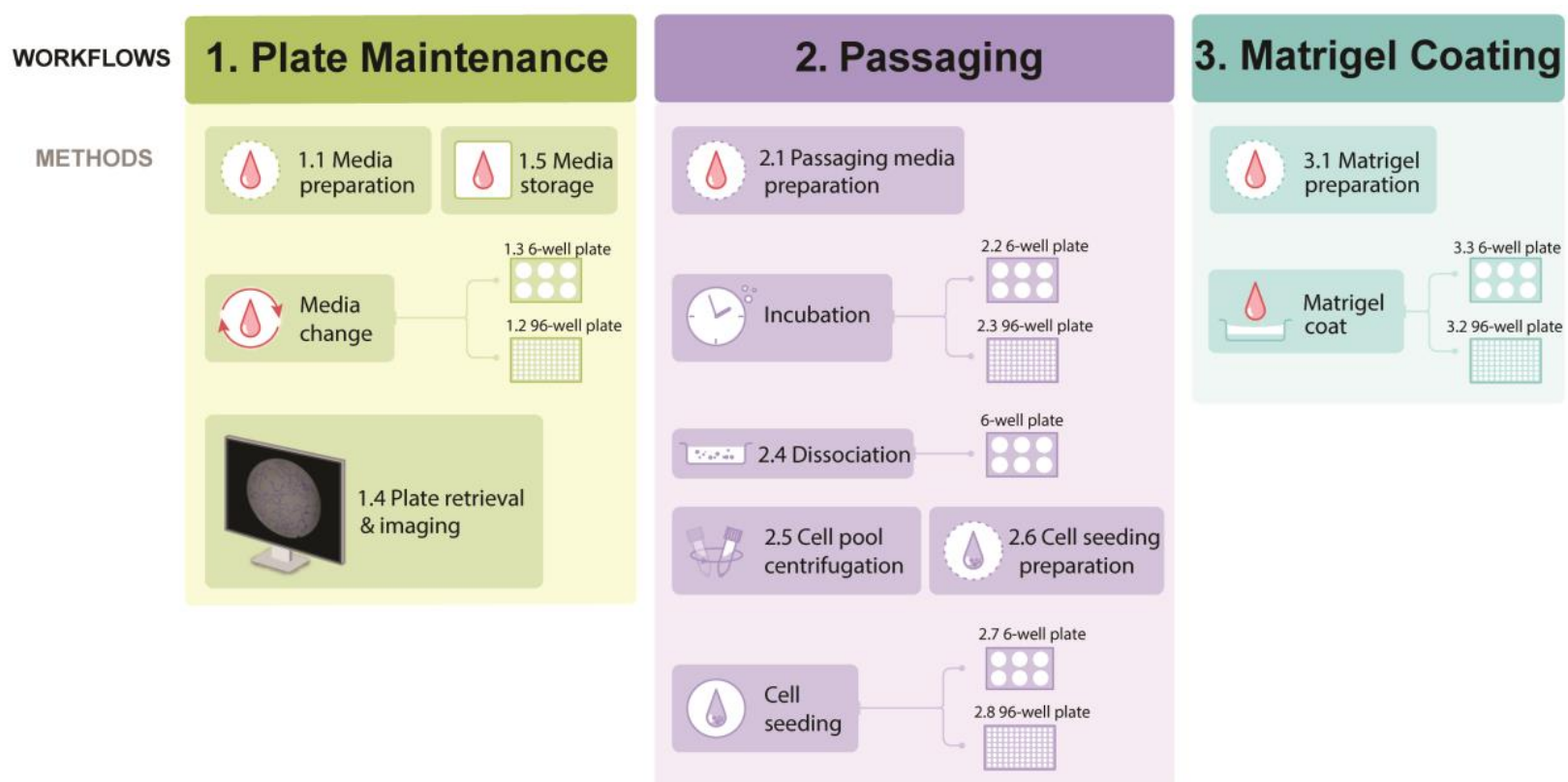

Figure 2. Graphical representation of the daily cell culture workflows: The automated methods are split into three main workflows: 1. Plate Maintenance, 2. Passaging, and 3. Matrigel Coating. Each workflow has unique and modular methods that are run sequentially when a workflow is started. Methods within a workflow can also be disabled and skipped or isolated and run separately.

These daily operations comprised three main workflows, each consisting of individual automated methods: 1. Plate Maintenance, 2. Passaging, and 3. Matrigel Coating (Figure 2). Plate Maintenance includes the replacement of old media with fresh media for both 6- and 96well plates, and automated imaging by the Celigo Imaging Cytometer (Figure 2, Workflow 1). Each day, we run the Plate Maintenance method early in the morning, prior to personnel arrival to the laboratory. This timing allows plates to be ready for imaging when personnel arrives to the lab during normal work hours. The Passaging method (Figure 2, Workflow 2) consists of cell expansion and includes preparation steps such as warming the enzymatic cell dissociation reagent and media to room temperature (RT) and incubating Matrigel-coated plates at $37^{\circ} \mathrm{C}$ prior to dissociating the cells from a selected 6-well plate. This is followed by centrifugation of the dissociated cells and preparation of cell seeding solutions, utilizing a 24-well reaction microplate (Figure 3H). The method finishes with the removal of Matrigel from pre-coated and warmed 6and 96-well plates and subsequent seeding of cells. We run this method after the Plate Maintenance method, examining cell morphology from well overview images (Figure 2, Method 1.3) to select wells of the 6-well plate that have met the QC criteria for passaging (see exclusion 
Automated hiPS cell culture

Allen Institute for Cell Science

criteria descriptions in Experimental procedures). We passage two duplicate 6-well plates in tandem, so that we have two plates to examine and choose from for subsequent cell passaging. The Matrigel Coating workflow consists of the mixing of the Matrigel solution, and the coating of 6- and 96-well plates (Figure 2, Workflow 3). This method is run after our Passaging method in our daily workflow, to ensure sufficient Matrigel-coated plates for upcoming assays. Each method can be run separately from the overall workflow, and workflows can be scheduled to run at designated times of day, allowing for more flexibility and easier adaptation to other applications. Parameters within each method of the workflows can be tailored to fit adaptations, i.e., every method is customizable. The three workflows and associated methods, including information on timing, necessary equipment, and key action parameters, are detailed in the Supplemental Information outlined in this report (Table S3). Further, we have created a video tutorial to explain the automated tissue culture platform, including movies of the robotic steps outlined in this report. The video tutorial can be accessed with this link: https://youtu.be/C KujKLS1wc.

\section{Key parameters for success}

Critical details of the automated cell culture methods were optimally tuned to ensure robust and consistent cell quality. This iterative process was laborious but necessary for success. The key parameters for each method of all workflows are listed in Table S3 and illustrated in Figure 3. They include the speed of aspirations, dispenses, and mixes of cells and solutions. For example, fine-tuning the rate at which mixing occurs during the resuspension of the cell pellet helps prevent stress-induced spontaneous differentiation and cell death while still achieving a single cell suspension. Further, a fast dispense of Matrigel to 6-well plates is crucial to ensure the entire well surface is coated. Other steps where aspiration and dispensing speeds are important are the dissociation and seeding of cells. For more information regarding the aspiration, dispense, and mix speeds are in Table S3.

Another key parameter is the location and depth of the tips in the wells, ensuring uniform distribution of both cells and solutions. During the aspiration of the supernatant, positioning the tips away from the cell pellet is important to ensure cells are left undisturbed. Using multiple tip positions while resuspending the cell pellet is also key as it gently and rapidly creates a single cell suspension (Figure 3G). This is also true for cell trituration and cell seeding (Figure 3A\&B), where up to eight different tip positions are used to dispense the cell suspension or solution in each large well of a 6 -well plate to achieve an even distribution. For smaller wells like those of a 96-well glass plate, the key issue is preventing the dislodgement of the Matrigel coating from the bottom of the well during cell seeding and media change. In this case, a single tip position to the side of the well leaves the Matrigel undisturbed which relates to more uniform cell seeding and growth (Figure 3D). Tip location is also important for Matrigel coating, where tips are strategically positioned so Matrigel evenly coats the surface of the 6- and 96-well plates (Figure 3D\&E).

Other key optimizations include reducing the speed of plates and reservoir movement across the deck to prevent spills and keep cells undisturbed. This speed can be reduced up to $25 \%$ of the default speed $(40 \mathrm{~cm} / \mathrm{sec}$ ) to mimic slower movements performed by cell culture experts (See Table S3). Further, the angle of inclination of a plate controlled by the tilt module (Figure 1 \#3-4; Table S2 \#3-4) replicates the manual tilting of tissue culture plates used by experts to prevent disturbing the cells during media aspiration. Steps utilizing tilt modules are outlined in Table S3. Finally, the temperature of cells and solutions controlled by the Cold Plate Air Cooled (CPAC) module (Figure 1 \#6-7; Table S2 \#6-7) are also critical to enhance enzymatic reaction for cell dissociation and to minimize stress to the cells during media change. The optimized speed values, angle of inclination and temperatures are listed in Table S3 for each step. The optimized relative positions for the tips within each well are illustrated in Figure 3. Additionally, all code for the described workflows and methods can be found at the following GitHub repository: (https://github.com/AllenInstitute/aics-automated-cell-culture-workflow). 
bioRxiv preprint doi: https://doi.org/10.1101/2020.12.18.423371; this version posted December 19, 2020. The copyright holder for this preprint (which was not certified by peer review) is the author/funder. All rights reserved. No reuse allowed without permission.

\section{Automated hiPS cell culture}

Allen Institute for Cell Science

A

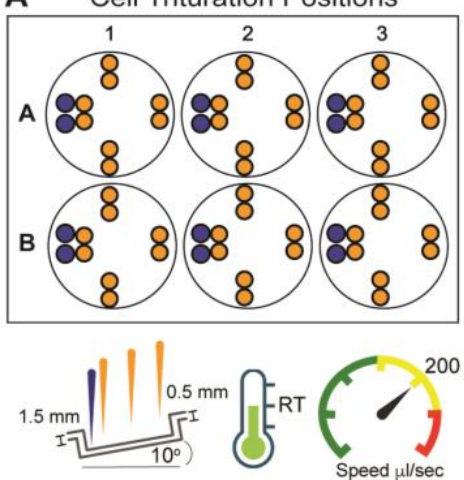

D
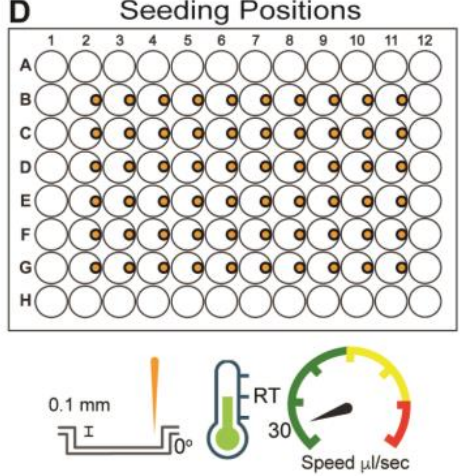

B

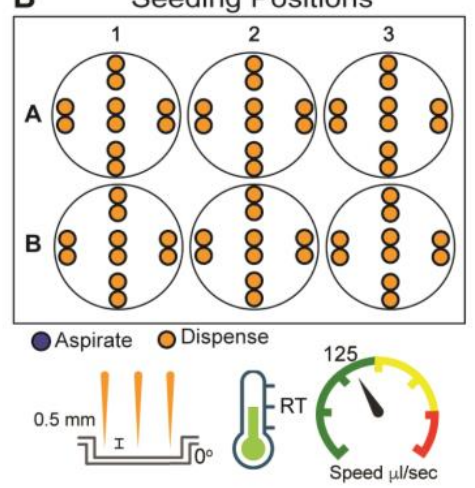

E Matrigel Coating Positions
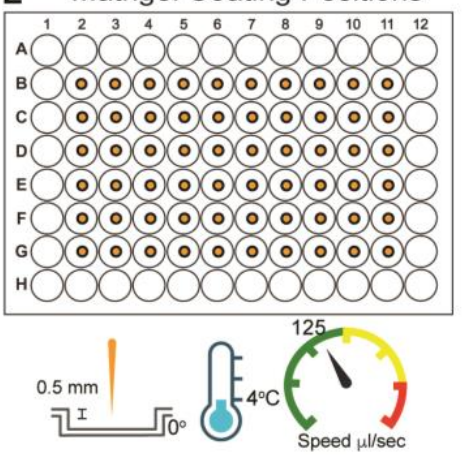

C Matrigel Coating Positions

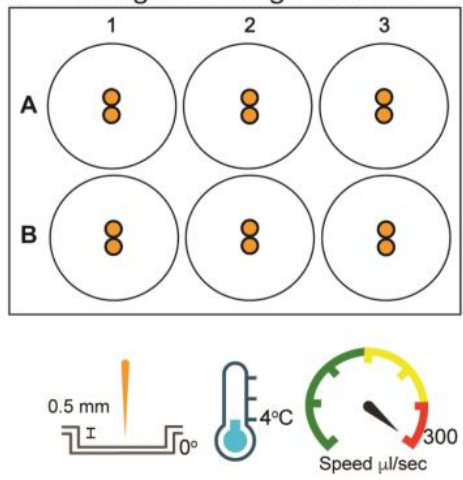

$\mathbf{F}$

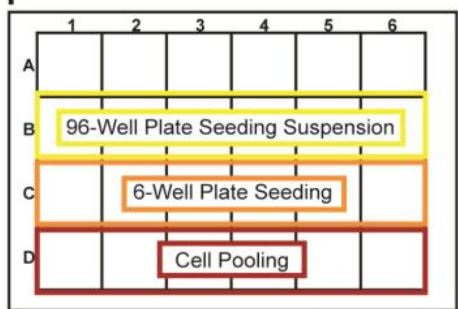

G

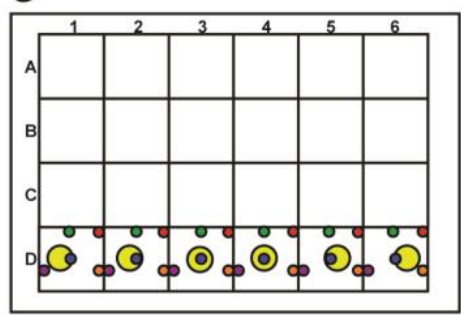

O Media Dispense Position 1

O Media Dispense Position 2

O Media Dispense Position 3

- Mix Position

O Supernatant Aspiration Position

Cell Pellet Location

Figure 3. Key action parameters: (A-E) Tip locations within plates; symbols below plates, from left to right, indicate the associated tilt angle, temperature, and speed of aspiration (blue circles and tips) or dispense (orange circles and tips) associated with each step. (A) Tip locations within wells of 6-well plates for cell trituration, (B) cell seeding, and (C) Matrigel coating methods. (D) Tip locations within wells of 96-well plates for media dispensing, cell seeding and (E) Matrigel Coating methods. (F) Row designations of the 24-well microplate for the centrifugation step of the passaging method. Row $B$ is for the 96-well plate seeding suspension, row $C$ is for the 6-well late seeding suspension, and row $D$ is for the cell pooling. (G) Tip locations within wells of the 24-well microplate; for the aspiration of supernatant (red), the mixing of cell suspension (blue) and for the media dispense (orange, green and purple). The cell pellet formed after centrifugation is shown in yellow. (H) Passaging of six unique cell lines in parallel, with each cell line as a different color. 
Automated hiPS cell culture

Allen Institute for Cell Science

Cells are grown and passaged in 6-well plates, collected, pelleted and resuspended using the 24-well microplate, and seeded in 6-well plates for expansion or 96-well plates for 3D high resolution imaging.

\section{Image processing workflow development}

Image processing pipelines were developed to estimate cell count and automatically select wells with best colony and cellular morphologies for subsequent 3D high resolution imaging (Figure 2, method 1.4; Table S3). Originally, 2D well bright field overview images from both 6- and 96-well plates were visually inspected and sorted by experts assessing cell health, colony growth, and confluency to identify problematic morphologies associated with cratering, colony lifting, cell differentiation and death (Figure 5D-G). Specific imaging QC requirements can be found in the Experimental procedures section below.

\section{Automated cell count from 6-well plate confluency}

The automated cell culture platform still required an operator to collect a sample of the cell suspension and run it on a cell counter to determine the volume of solution needed for the desired cell seeding density. We eliminated this manual step through an image processing pipeline using the bright field well overview images from 6-well plates to estimate, from the well confluency, the total number of cells (Figure 4A-E). The image processing pipeline was composed of six main steps, performed on individual tiles of the overview image (Figure 4A\&B): Well edges are masked (i.e. masking out air bubbles and well edge skirt), the edges are enhanced with a Sobel filter (Scikit image, (Walt et al., 2014)), and the images are then segmented into colony and background areas. Segmentation artifacts are resolved using image processing methods such as erosion, dilation and filling holes of segmented objects. Finally, colony areas are measured, summed up for all tiles of a given well to calculate well confluency (Figure 4B) and confluency measurements saved to a database. The 6-well CellProfiler image processing pipeline generates the confluency from a well by summing up the colony area of each tile divided by the total area in a well (Figure 4C). A robust positive relation exists between well confluency and cell counts (Figure 4D) with Pearson correlation of $0.88(n=19)$, and bootstrapped median $R^{2}$ of 0.74 . We validated this approach by comparing the confluency of wells after 4 days post seeding using the estimated cell counts from the regression model and that of a cell counter and found comparable cell morphology and confluency between wells of 6-well plates (Figure 4A \& 5A) and wells of 96-well plate using both methods (Figure 4E). The results show that it is possible to predict cell count from confluency measurements and the data can then be used to estimate the volume of media in which cells should be resuspended and seeded.

\section{Automated 96-well plate and well selection}

To eliminate the need for experts to subjectively select wells for high resolution imaging, we developed an image processing pipeline using a combination of llastik pixel classifier (Berg et al., 2019) and CellProfiler (Carpenter et al., 2006; Kamentsky et al., 2011; McQuin et al., 2018) for automated well selection. The llastik and CellProfiler pipelines are available for download from GitHub (https://github.com/AllenInstitute/aics-automated-cell-culture-workflow). The llastik pixel classifier (which defines smooth and rough pixels) and CellProfiler pipeline (for colony segmentation) allowed us to discriminate regions of morphology in colonies considered problematic, at a higher accuracy than standard per object-based measurements. In addition, this combination allowed for a higher detection rate of fully lifting and cratering colony morphologies but was unable to detect partial lifting colony areas. 
bioRxiv preprint doi: https://doi.org/10.1101/2020.12.18.423371; this version posted December 19, 2020. The copyright holder for this preprint (which was not certified by peer review) is the author/funder. All rights reserved. No reuse allowed without permission.

\section{Automated hiPS cell culture \\ Allen Institute for Cell Science}

Cell Count from Confluency

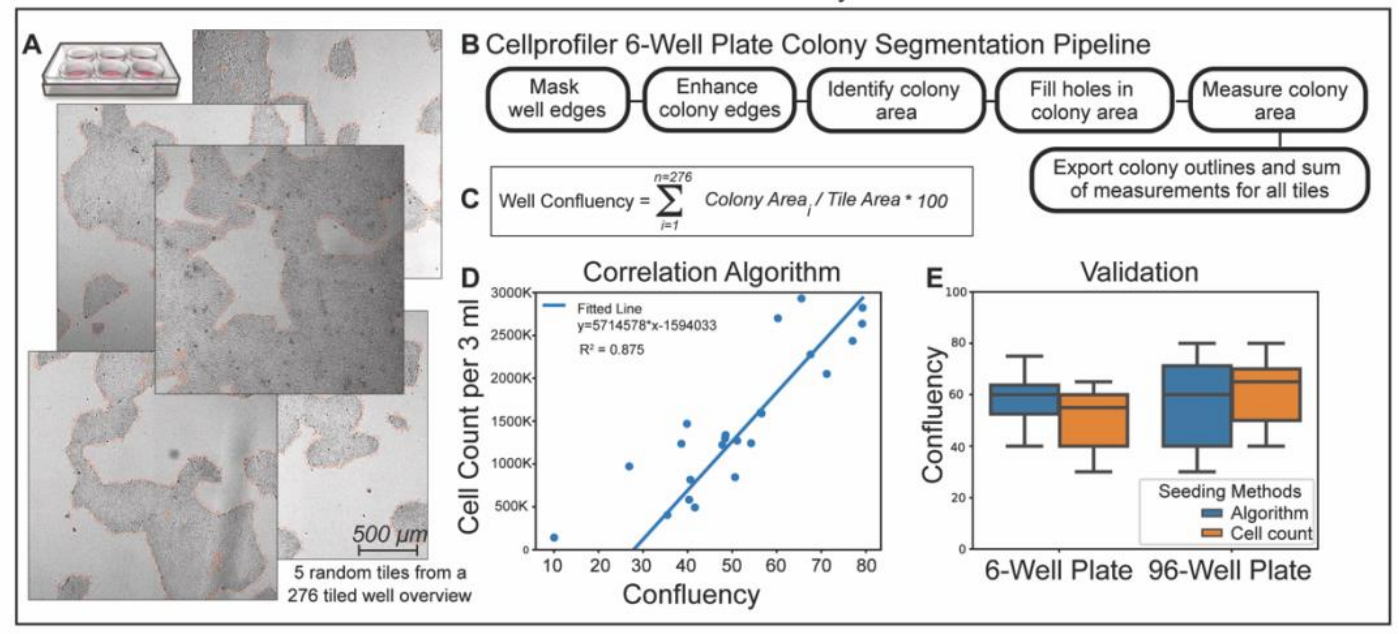

Automated Well Quality Control and Ranking

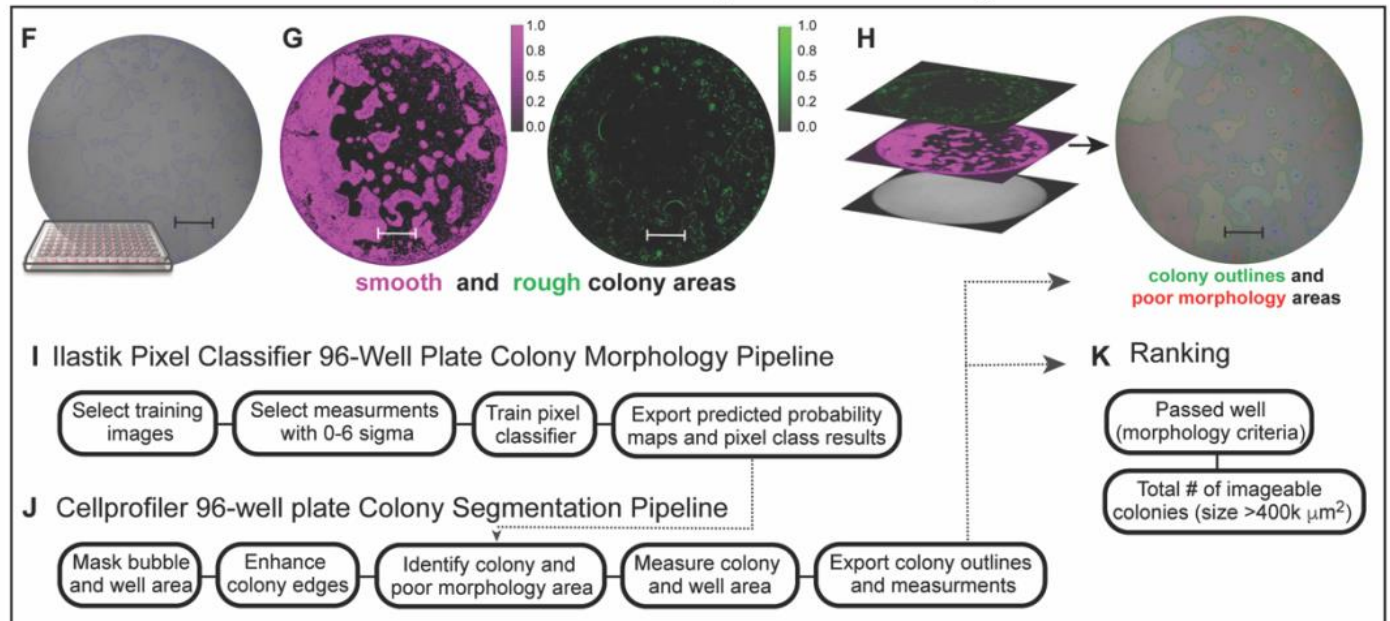

L Validation of Automated Well Classifier Performed on 205 Well Overview Images
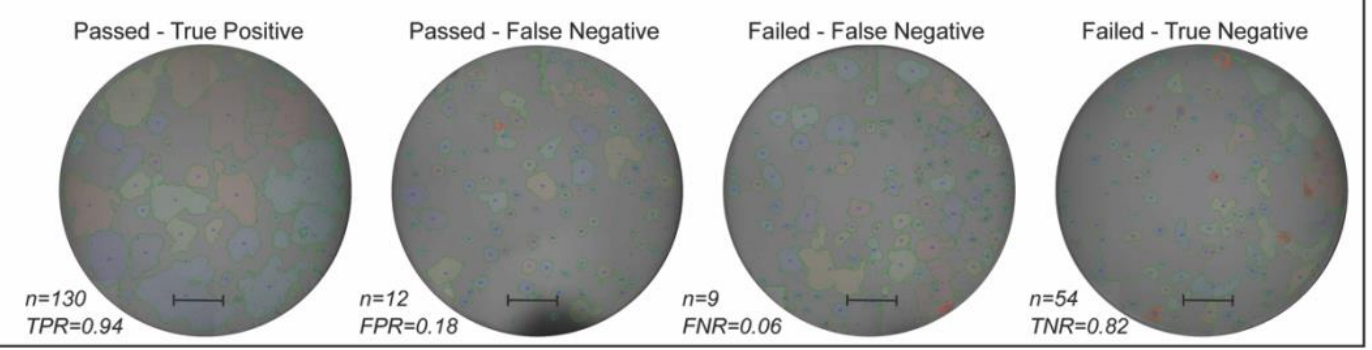

Figure 4. Automated image processing to calculate well confluency, cell counts, and imaging well selection:

(A) Brief schematic of 6-well plate with segmentation tiles (unstitched) outlining the colony area. Scale bar is $500 \mu \mathrm{m}$ (B) Images acquired from a Celigo imaging cytometer were processed to segment and identify colonies in the wells and calculate colony features. (C) Formula used to calculate the well confluency. (D) Cell count numbers obtained from the cell counter were plotted against well confluency and shows positive correlation between the variables. (E) Boxplot of confluency of 6-well and 96-well plates seeded based on cell-count (orange) or on estimate from algorithm (blue). (F) An example of segmented well image from a 96-well plate. (G) An example of the smooth and rough pixel probability output channels from the llastik pipeline. (H) An example of the CellProfiler image inputs: bright field and the rough/smooth pixel probability channels. (I) Steps of the llastik pixel classification pipeline. (J) Steps of the CellProfiler colony segmentation and measurement pipeline. Colony segmentation overlay output image with poor morphology regions outlined in red. (K) Passed well filtering and ranking of total number of imageable wells per plate. (L) Validation with labeled data of the automated plate scoring pipeline. Other well level aggregation metrics such as average number 
Automated hiPS cell culture

Allen Institute for Cell Science

of colonies per cell line, average colony area per cell line, and average confluency are useful for assessing plates within an imaging date batch. Well scoring classifier metrics: True positive rate (TPR) 0.94 , false negative rate (FNR) 0.06 , true negative rate (TNR) 0.82 , false positive rate (FPR) 0.18 , positive predictive value (PPV) 0.92 , negative predictive value (NPV) 0.86 , accuracy (ACC) $0.90, \mathrm{~F} 1$ score 0.93 . All scale bars are $1 \mathrm{~mm}$ unless otherwise specified.

Using the measurements and features extracted by the llastik pixel classifier (Figure 4G\&I) and CellProfiler pipeline (Figure 4F\&J) combination, we developed an automated, robust, quantitative and unbiased method for the selection and ranking of wells for imaging at high resolution. The criteria for selecting wells for imaging at high resolution are: 1) Confluency between $40 \%$ and $85 \%, 2$ ) three or less colony morphology issues (cratering, partial and complete lifting) per well, 3) at least three colonies per well greater than $400,000 \mu^{2}$. We validated this by comparing the percent level of agreement between the expert selection and the ranking list. Wells that passed morphology criteria (llastik pixel classifier, Figure 4I) were sorted by cell lines and according to the total number of colonies greater than $400,000 \mu \mathrm{m}^{2}$; wells that were outside the confluency range were filtered out (CellProfiler, Figure $\mathbf{4 K}$ ). The validation of the automated classification of wells into a pass or fail score was compared to that of expert annotators which displayed a high level of agreement (positive predictive value (PPV) 0.92, negative predictive value (NPV) 0.86 , accuracy (ACC) 0.90 , F1 score 0.93 ) with a true positive rate (TRP) of 0.94; Figure $4 \mathrm{~L}$ ). Even with a false positive rate (FPR) of 0.18 , wells from this group were seldom found within the top 40 of the ranked daily 300 wells and thus are unlikely to be imaged at high resolution. This ranking list is the final step providing a fully automated cell culture and imaging plate production for our imaging pipeline.

\section{Automated cell culture method validation}

To optimize each parameter and validate that the automated cell culture method produced equivalent hiPSC quality to that of the manual cell culture method we compared side-by-side the colony and cell morphology, expression of stemness markers, and cytogenetic stability of cells produced by both methods. The first step was to scale down the cell culture conditions, comparing the results of passaging hiPSCs manually in $10 \mathrm{~cm}$ dishes to that of passaging them manually in 6 -well plates. We then compared the results of passaging cells in 6 -well plates with the manual and automated methods. We also seeded cells passaged by both methods into glass bottom 96well imaging plates, to verify the quality of imaging assay plates produced from different culturing methods.

\section{Morphology characteristics assessment}

Cells were examined for stem-like features including monolayer morphologic homogeneity and colonies with smooth and defined edges (Roberts et al., 2017). Visual inspection showed no major morphological differences when observed at $2 \mathrm{X}$ and 10X magnifications (Figure 5A\&C). The frequency of undesirable morphologies, e.g., cratering (Schulz et al., 2003) (Figure 5G), complete or partial lifting of colony edges (Figure 5D\&F) and stretched cells at the colony edges (Figure 5E), were tallied among bright field images taken at a magnification of $2 \mathrm{X}$ across ten passages. We observed a low and comparable frequency of crater morphology in cultures from $10 \mathrm{~cm}$ dishes (7\%) and 6-well plates (4\%). Similarly, low and comparable frequencies of colonies with stretched edge cells were observed between the two type of plates $(7 \%$ and $8 \%$ respectively). No complete or partial lifting of colony edges was observed.

To ensure the automated method produced 96-well imaging plates of high quality for our imaging pipeline, experts scored 96-well plate overview images seeded with cells from 6-well plates passaged by both manual and automated methods over four separate passages. Expert assessments identified similar percentages of wells from the two methods meeting the QC 


\section{Automated hiPS cell culture}

Allen Institute for Cell Science

requirement for imaging (see QC descriptions in Experimental procedures) in 3D at higher magnification (Figure D-G\&K). Expert scoring was performed for a total of 240 wells from cells passaged with the manual method and 1,080 wells from cells passaged with the automated method of which $55.4 \%$ (133 well total) and $67.3 \%$ (727 well total) passed the imaging QC requirements, respectively (Figure $5 \mathrm{~K}$ ).

Figure 5. Cells cultured with the manual and automated methods present similar quality control characteristics:

A
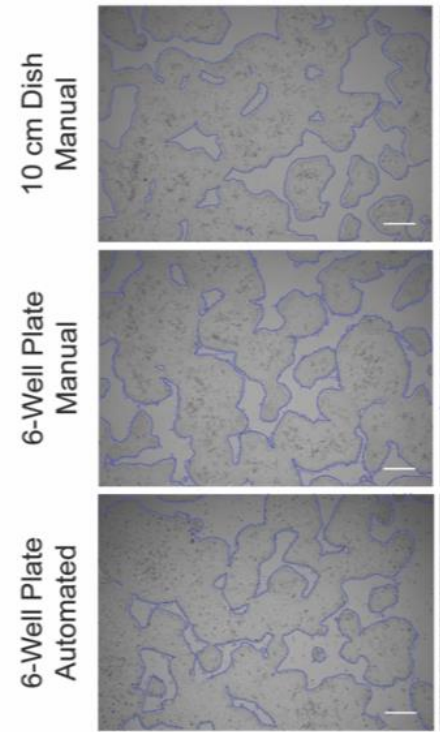
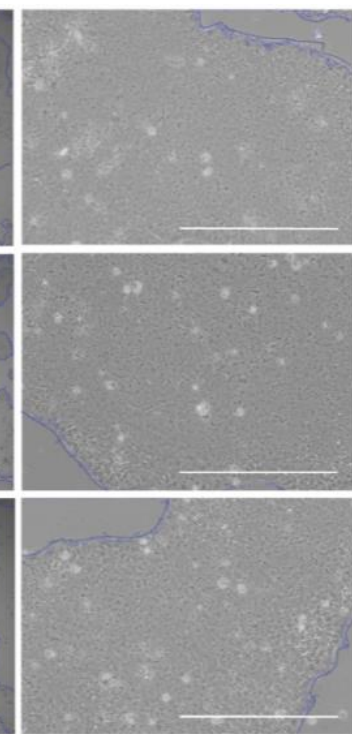

B

\begin{tabular}{|c|c|c|c|c|}
\hline & \multicolumn{3}{|c|}{ Stemness markers, $\%$ positive } \\
\hline Cell Lines & Method & Oct 3/4 & Nanog & SSEA4 \\
\hline \multirow{2}{*}{$\begin{array}{c}\text { AICS-0 } \\
\text { (Unedited WTC) }\end{array}$} & Manual & 98.8-99.3 & $99.2-99.6$ & 100 \\
\hline & Automated & 97.8-98.4 & $97.5-98.6$ & 99.8-99.9 \\
\hline \multirow{2}{*}{$\begin{array}{c}\text { AICS-54 } \\
\text { (AAVS1 cl. 91) }\end{array}$} & Manual & $99.1-99.2$ & $99.3-99.4$ & 99.9-100 \\
\hline & Automated & $\begin{array}{l}8.5-98.8 \\
\end{array}$ & $98.8-99.0$ & 99.9-100 \\
\hline
\end{tabular}

C 96-Well Plate Manual
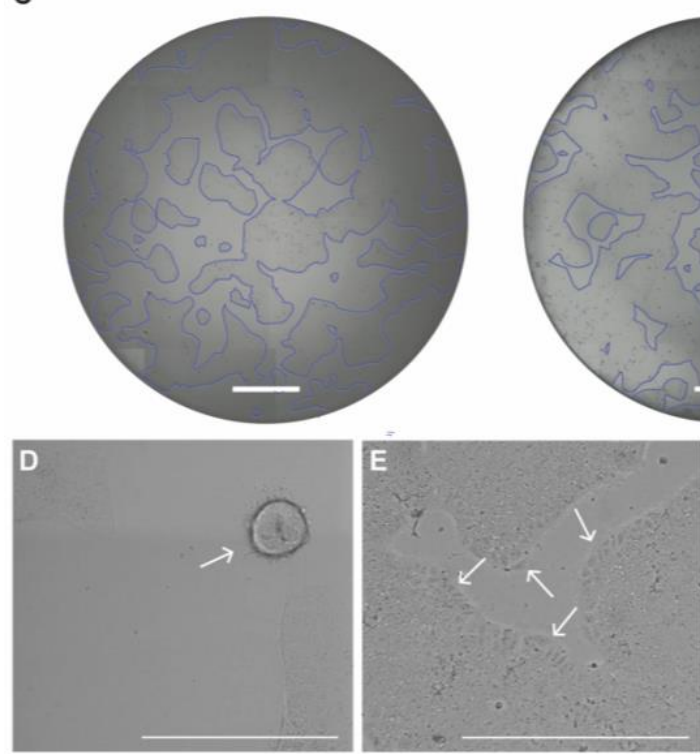

H

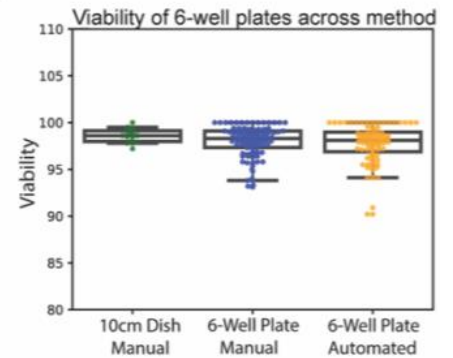

I
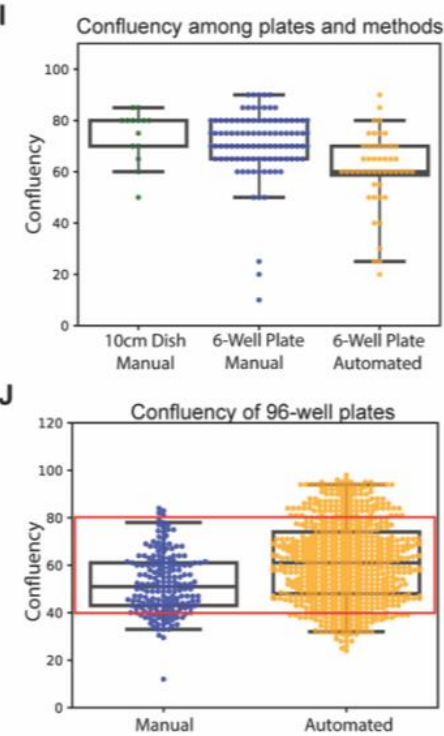

K
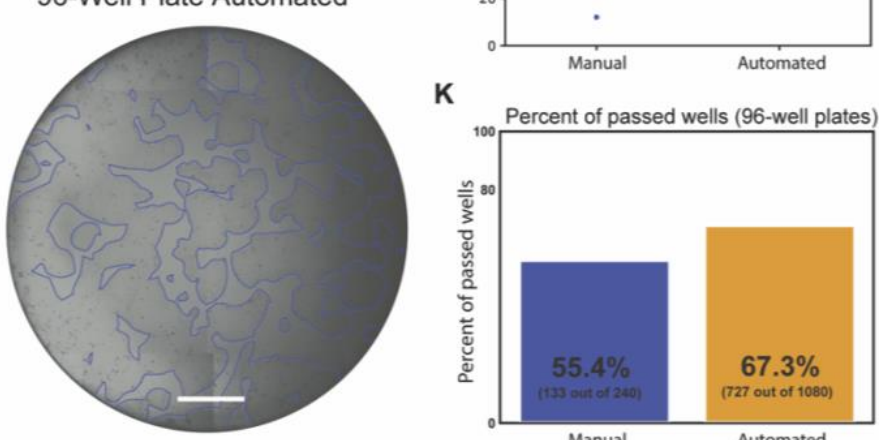

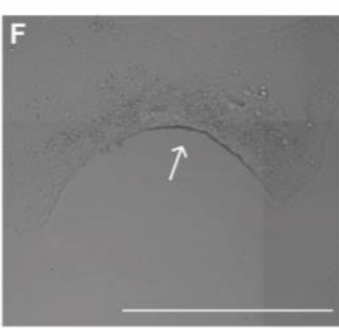

Manual Automated

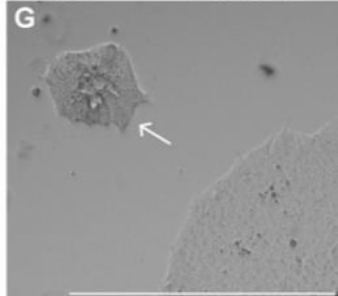

(A) Representative images of cells (2X and 10X magnifications) passaged in $10 \mathrm{~cm}$ dishes via the manual method and 6 -well plates via both the manual and automated methods. Scale bars are $500 \mu \mathrm{m}$. (B) Table showing the percentage 
Automated hiPS cell culture

Allen Institute for Cell Science

of cells expressing surface and intracellular stemness markers as indicated by flow cytometric analysis. Values range across $>3$ experiments. (C) Representative overview well images from 96-well glass plates from cells passaged and seeded with the manual (left) and automated (right) methods. Scale bars are $1 \mathrm{~mm}$. (D-G) Representative images of undesirable colony characteristics that can be observed during morphological assessment. Arrows point to the region of the colony with specific morphological characteristic such as: (D) colony fully lifted, (E) stretched cells at the edges of colonies, (F) colony that has partially lifted and, (G) crater within a colony. (H) Average percent of viable cells passaged in either $10 \mathrm{~cm}$ dishes (green), 6-well plates manual (blue) or automated (orange) methods. (I) Average percent confluency of cells passaged in either $10 \mathrm{~cm}$ dishes (green), 6-well plates with manual (blue) or automated (orange) methods. (J) Average percent confluency per well of 96-well plate passaged either with manual (blue) or automated (orange) methods. Red box represents the well percent confluency range meeting imaging QC requirements (K) Histogram indicating the percent of wells from 96-well imaging plates that met imaging QC requirements from cell passaged with either the manual (blue) or automated (orange) methods. Imaging QC requirements (passed wells) are that a well has $<3$ instances of cratering, $<3$ instances of full or partial colony lifting, and percent confluency within $40-$ $80 \%$.

\section{Growth, Stemness marker expression and cytogenetic analysis}

The percent confluency for $10 \mathrm{~cm}$ dishes, 6-well and 96-well plates and percent cell viability for $10 \mathrm{~cm}$ dishes and 6-well plates were also evaluated by experts and analyzed across 10 passages; there were no significant differences among the different cell culture plates $(P=0.23$ and $\mathrm{P}=0.26$ respectively; Figure $5 \mathrm{H} \& \mathrm{I})$. Similarly, among 6 -well plates passaged with either the manual or automated methods, no significant difference in the percent of viable cells was observed ( $\mathrm{P}=0.32$; Figure $\mathbf{5 H})$. All methods and tissue culture plate types had average percent cell viabilities that were above our threshold requirement $(10 \mathrm{~cm}$ dish $(98.6 \%)$, manual 6 -well plate $(98.0 \%)$, and automated 6-well plate $(97.7 \%))$. Finally, $72 \%$ of the wells from a 96 -well plate seeded with cells passaged with the automated methods had a percent confluency meeting the QC requirement compared to $83 \%$ of wells from the manual method (Figure $5 \mathbf{J}$ ). Finally, although the percent of wells of a 96-well plate seeded with the manual method was found to be slightly higher (83\%) than that of wells seeded with the automated method $(72 \%)$, the actual number of wells meeting the imaging QC requirements is much higher for wells seeded with the automated method (488 wells), than seeded with the manual method (152 wells) over the same time frame (four passages) due to the increased throughput of the automated tissue culture platform (Figure $5 \mathrm{~K})$.

Oct3/4, Nanog, and SSEA4 expression were used to assess stemness of the cultures (Baghbaderani et al., 2015). Both methods demonstrated high pe of stemness marker expression at both passages 8 and 15, with the percent of positively fluorescent cells ranging from $97.5-100 \%$ across all stemness markers, cell lines and passage methods (Figure 5B). Cytogenetic stability determined by quantifying G-band karyotypic abnormalities also did not differ between automated and manual cell culture methods.

\section{Single cell organization comparison}

To determine whether the automated and manual cell culture methods give rise to differences in cellular organization in 3D high resolution images, live cells cultured with either method were imaged on spinning-disk confocal microscopes after membrane (CellMask Deep Red) and DNA (NucBlue Live) staining. Using an hiPSC line expressing mEGFP-tagged Tom20, which localizes in mitochondria, we observed no detectable morphological difference in the amount or localization of mitochondria between cells cultured with either methods (Figure 6A\&B).

Additionally, we compared the cell and nuclear shape by segmenting all cell in 3D using the Allen Cell Structure Segmenter (Chen et al., 2018) and calculated the cell and nuclear volume and sphericity for every cell. No significant difference between the manual and automated methods was observed in the average cell volume, which were $1,380 \mu \mathrm{m}^{3}(\mathrm{n}=179)$ and 1,426 $\mu \mathrm{m}^{3}$ 
Automated hiPS cell culture

Allen Institute for Cell Science

( $n=378$; Figure 6C), respectively. The nuclear volume was also unaffected by the automated cell culture method with an average volume of $373 \mu \mathrm{m}^{3}(n=378)$ compared to $368 \mu^{3}(n=179)$ for manually cultured cells (Figure 6D). Cellular and nuclear sphericity (index of cell flatness) in function of cell density (estimated as the number of cells within a field of view) and an index of cell packing) revealed no difference between the two methods (Figure 6 E-F).

Finally, using DNA staining, we quantified the number of mitotic cells for each condition. We found similar percentage of mitotic cells between manual $(4.7 \% ; n=1,426)$ and automated $(4.5 \% ; n=2,064)$ methods $(P=0.77)$. Altogether these results suggest that the automated cell culture method does not alter the shape of the cells nor their division rate.
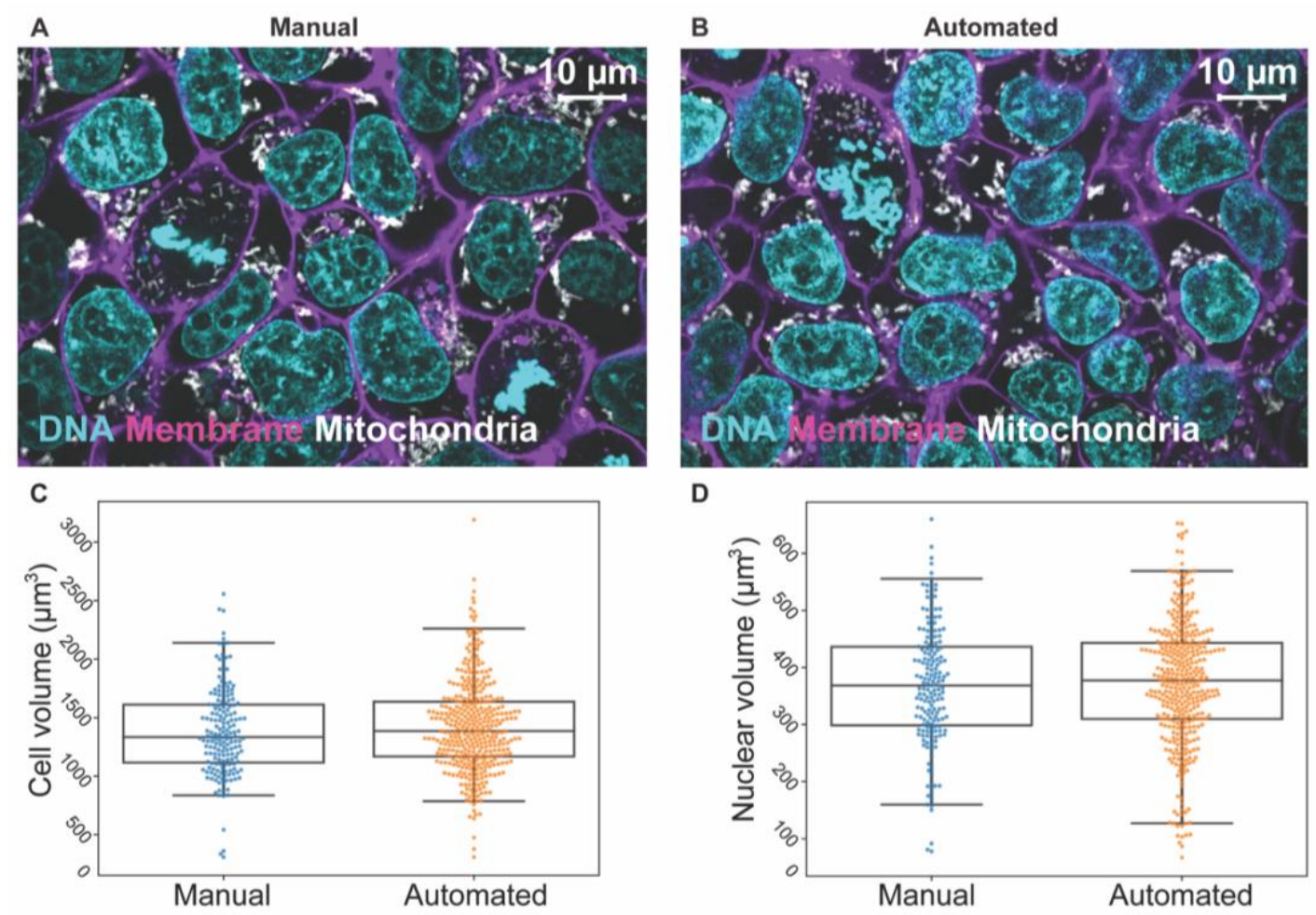

D
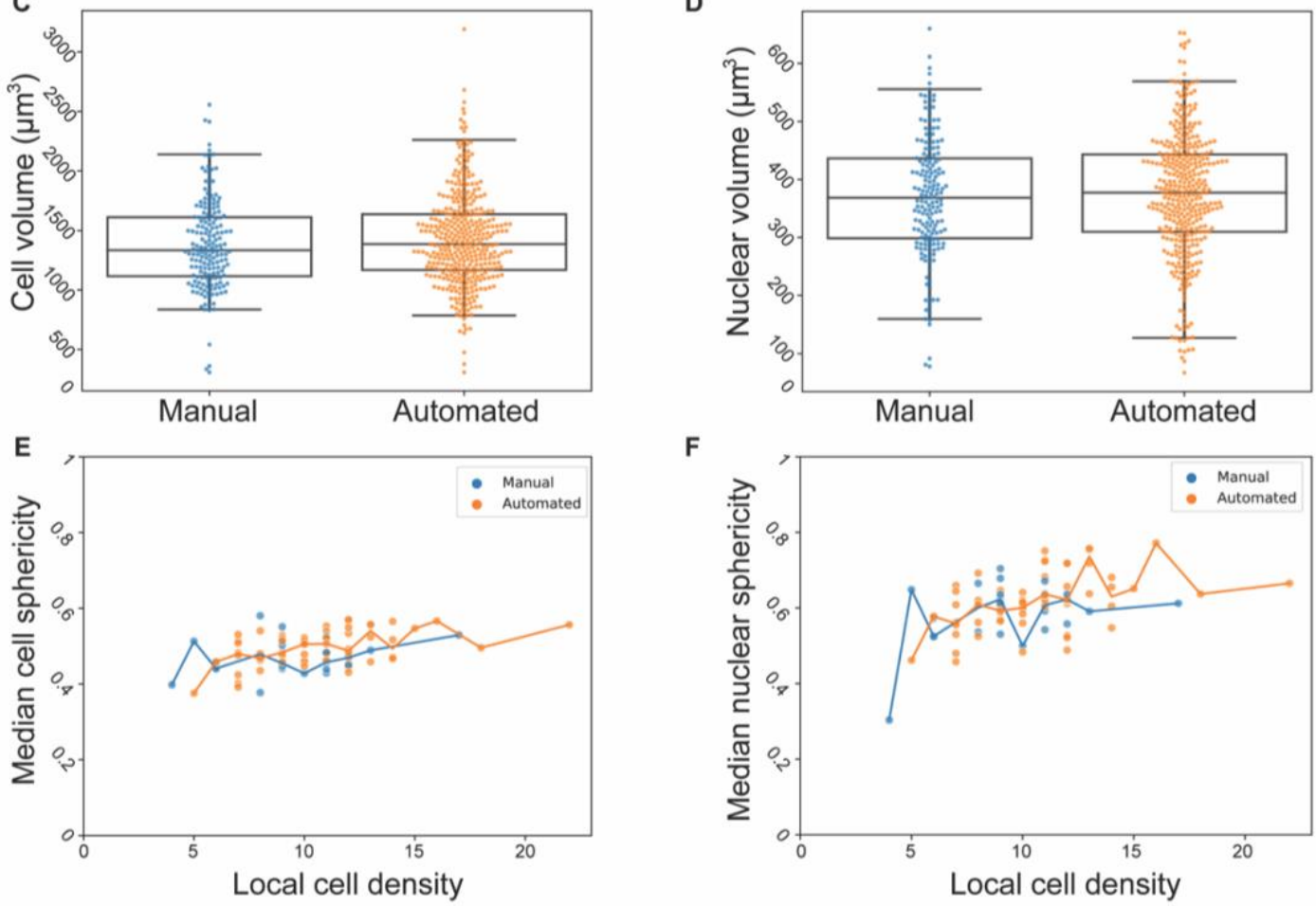

$\mathbf{F}$

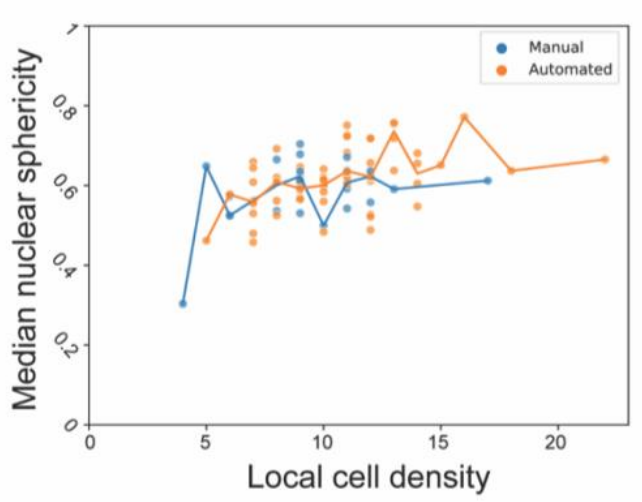

Figure 6. Cells cultured with the manual and automated methods present similar organizational and phenotypical characteristics: (A-B) Spinning-disk confocal images of one z-slice of mEGFP-tagged Tom20 expressing cells (mitochondria, greyscale) cultured with the manual or automated methods and stained for DNA (blue) and cell membrane (magenta). Scale bar is $10 \mu \mathrm{m}$. (C-D) Cell and nuclear volume for the cell cultured with the manual (blue) or automated (orange) methods. (E-F) Median cell and nuclear sphericity in function of the local cell density (number of cells in the field of view) for the cell cultured with the manual (blue) or automated (orange) methods. 
Automated hiPS cell culture

Allen Institute for Cell Science

\section{Discussion}

This method describes a workflow for optimizing and replicating an automated platform for high quality, high throughput hiPSC culture. The method produces results comparable to those from manually cultured hiPSCs. The modular workflow uses a liquid handling system customized for the automated passaging and seeding of gene-edited hiPSCs on optical grade glass bottom plates for high resolution 3D microscopy, increasing culturing standardization and reproducibility for imaging applications. More specifically, the automation ensured standardized and reproducibility of speeds at which cells and solutions are aspirated and dispensed, location of tips within wells, and timing within and between steps, all known to introduce experimental variability. The platform also eliminates the need for highly trained and experienced personnel to execute these tasks. Even with experts, variability in manual execution is unavoidable, making consistent reproducibility difficult to achieve.

Manual culturing of hiPSCs also requires daily involvement, including early morning, late evening, and weekend work. The ability to perform maintenance and passaging without requiring personnel to be on site is one of the main advantages of the platform. Automating the centrifugation and cell count steps also enables unattended weekend passaging and consistent four day spacing between each hiPSC passage. Another important advantage is the use of quantitative imaging to monitor the cells confluency and morphology at more flexible times of the day with minimum cell disruption. This last step, while not unique to our system (Haenel and Garbow; Jaccard et al., 2014), allowed us to eliminate the cell count from a cell suspension and to fully automate the platform without any need for attendance during the Passaging method steps of our workflow.

While we are not the first to develop a fully automated platform for hiPSC culture (Archibald et al., 2016; Baghbaderani et al., 2015; Crombie et al., 2017; Daniszewski et al., 2018; Kami et al., 2013; Koike et al., 2012; Paull et al., 2015; Terstegge et al., 2007; Thomas et al., 2009; Wang et al., 2014), to our knowledge, we are the first to report the automation of undifferentiated hiPSC culture on glass bottom plates compatible with 3D high resolution microscopy. Previous automated PSC culture platforms used traditional plastic dishes (Hussain et al., 2013; Konagaya et al., 2015; Terstegge et al., 2007; Thomas et al., 2009) known to provided better attachment and growth for cells but limiting the quality of the images acquired with high resolution 3D microscopy (Webb and Brown, 2013). Additionally, others who also compared cell characteristics, e.g., morphology and gene expression between manually and automatically cultured cells have also shown that automating passaging of pluripotent stem cells creates more uniformed cells than those manually cultured (Daniszewski et al., 2018).

Importantly, we have compiled and reported sufficient details to provide a turn-key automated system for the culture of undifferentiated hiPSCs that can be readily replicated in other labs. While some platforms were developed for the large scale production and biobanking of iPSC lines from donors with a wide range of diseases (Thomas et al., 2009), others have emphasized the automation of differentiation protocols (Chatterjee et al., 2015; Crombie et al., 2017; Hussain et al., 2013; Le and Hasegawa, 2019; Paull et al., 2015) or reprograming protocols (Baghbaderani et al., 2015; Chan et al., 2020; Conway et al., 2015; Paull et al., 2015); but few described every element of the automation platform, providing access to all programmatic files used to run the different methods. By doing so we hope others can easily replicate this platform in total or in part with similar equipment. 
Automated hiPS cell culture

Allen Institute for Cell Science

The success of this platform relied on the optimization of several key parameters. For example, by reducing the speed of movements we prevented spills. By optimizing the aspiration and dispense speeds of cells and media, in addition to the number of cells line passaged in parallel, we prevented leaving cells in suspension or without media for longer periods of time than recommended. By optimizing the location of the tips in wells and speed of aspiration and dispense, we prevented disturbing the cells and improve cell mixing and dispersion. We also saved time and improved consistency of our cultures by utilizing standardized plate inclination, heating and cooling temperatures and storage and incubation times for all reagents. Determining a consistent number of plates to use in tandem, along with the type of cell culture plates to use also improved reproducibility of our imaging samples.

In developing this platform, we encountered two important challenges. The first was to successfully coat 96-well glass bottom plates with Matrigel. Many adaptations were required to ensure Matrigel was coating and adhering to the entire surface of each glass bottom well uniformly. Matrigel coating, a minimum overnight incubation at $4^{\circ} \mathrm{C}$ in addition to a $2 \mathrm{hr}$ incubation at $37^{\circ} \mathrm{C}$ prior to removal of Matrigel and seeding of cells, was crucial for optimal Matrigel adhesion to the glass substrate, and consequent colony adhesion and cell growth. The second challenge was maintaining the stem-like morphology of hiPSCs seeded on optical grade glass surfaces. The Matrigel coating optimization step turned out to be key to the preservation of the stem-like morphology of cells seeded on glass surfaces. Additionally, other steps, including 'gentle' aspiration and dispense speeds, slowed movements of plates containing cells, and minimized time outside of the incubator, prevented stress-induced spontaneous differentiation.

A major application of our automated cell culture pipeline is to produce standardized imaging samples for a microscopy pipeline. Our automated method allows us to produce a large number of wells per day that meet imaging QC requirements. One round of passaging and seeding can produce over 300 wells from 96-well plates for high resolution imaging, facilitating productivity, imaging capability and pipeline efficiency. Although much of our cell culture pipeline is automated, a few human interventions remain. For example, the thawing of source cells is still performed manually. After 96-well imaging plates have reached four days of growth, and are deemed ready and suitable for imaging, they must be manually moved from the $37^{\circ} \mathrm{C}$ incubator and placed onto the spinning-disk microscope. Further logistical steps that require human interaction include the filling of reagent reservoirs and subsequent placement in $4{ }^{\circ} \mathrm{C}$ refrigerator, the re-loading of $1,000 \mu \mathrm{L}$ tips and $300 \mu \mathrm{L}$ tips and responding to robotic errors that cannot be fixed remotely. Further automation and expansion of this platform could be achieved with equipment like a decapper, an articulating arm, and an automatic tip loader, further eliminating the need for manual interactions.

With this publication, we intend to share as many details as possible for others to replicates and build their own automated culturing platforms. By including details, visuals, and action parameters, we intend to facilitate a smoother transition from manual to automated hiPSC culture, increasing reproducibility and throughput and decreasing user fatigue implicit to the manual approaches. 


\section{Experimental Procedures}

\section{Cell lines}

The handling methods for hiPSC lines was internally approved by an oversight committee and all procedures performed in accordance with the National Institutes of Health, National Academy of Sciences, and Internal Society for Stem Cell Research guidelines. The gene edited cell lines used in this study were created using the parental Wild Type (WTC-11) hiPSC line, derived from a healthy, male donor (Kreitzer et al., 2013). Each gene-edited cell line harbors a fluorescently tagged protein localizing to a cellular structure. The CRISPR/Cas9-mediated genome editing methodology used to generate these cell lines was described in (Roberts et al., 2017). The identity of the unedited parental line was confirmed with short tandem repeat (STR) profiling testing (29 allelic polymorphisms across 15 STR loci compared to donor fibroblasts). Since WTC-11 is the only cell line used by the Allen Institute for Cell Science, edited WTC-11 cells were not re-tested because they did not encounter other cell lines. Cell lines are described at https://www.allencell.org and are available through Coriell at https://www.coriell.org/1/AllenCellCollection. For non-profit institutions, detailed MTAs each cell lines are listed on the Coriell website. Please contact Coriell regarding for-profit use of cell lines as some commercial restrictions may apply.

\section{Cell culture reagents}

Single cavity reservoirs (Agilent) were used to store reagents in the Cytomat 6002 and on the STAR deck. Clear-skirt, sterile, 6-well plates (Greiner Bio-One) and glass bottom, black-skirt, 96-well plates with \#1.5 optical grade cover glass (Cellvis) were coated with GFR Matrigel basement membrane matrix, phenol red-free (Corning) stored in 2-column reservoirs (Agilent) and diluted with Dulbeco's modified eagle medium (DMEM)/F-12 (1X, Thermo Fischer Scientific). Cells were collected in a 24-well microplate (Agilent). Lids (Agilent) were used with all reservoirs. Conductive tips for the STAR included $1,000 \mu \mathrm{L}$ filter tips and $300 \mu \mathrm{L}$ nested tips without filters (Hamilton Company). Cells were cultured in mTeSR1 medium with and without phenol red (STEMCELL Technologies Inc.), supplemented with 1\% penicillin-streptomycin (Thermo Fischer Scientific) and, for $24 \mathrm{hrs}$ post-seeding with $10 \mathrm{mM}$ Rho-associated protein kinase (ROCK) inhibitor (Y-27632, STEMCELL Technologies Inc.). Dulbecco's Phosphate-Buffered Saline (DPBS) $1 \mathrm{X}$, and StemPro Accutase cell dissociation reagent (all Thermo Fisher Scientific) were also used. Cell counting was performed with a Vi-CELL XR Series cell viability analyzer and associated Vi-CELL XR sample vials (Beckman Coulter). Table S1 contains a complete list of cell culture reagents and consumables.

\section{Equipment}

The automated cell culture platform was developed on a Hamilton Microlab STAR Liquid Handling System (Hamilton Company) (Table S2). Integrated storage components include a Cytomat $6002\left(4^{\circ} \mathrm{C}\right.$ refrigerator; Figure 1 \#22; Table S4, \#22) and Cytomat $24\left(37^{\circ} \mathrm{C}\right.$ incubator; Figure 1\#21; Table S4 \#21) (Thermo Fischer Scientific), each equipped with a plate shuttle system. Also integrated are the Microplate Centrifuge (Agilent; Figure 1\#20; Table S4 \#20) and for low magnification, automated imaging, the Celigo Imaging Cytometer (Nexcelom; Figure 1 \#19; Table S2 \#19). For handling of labware on the STAR deck and between integrated components, the Internal Swivel Arm Plate (iSwap) Handler component and Compressed O-Ring 
Automated hiPS cell culture

Allen Institute for Cell Science

Expansion (CO-RE) gripper paddles are used (Table S2). The STAR has an eight-channel arm ( $1 \mathrm{~mL}$ capacity/channel) for pipetting, two CPAC heating and cooling stations (Figure 1\#6-7; Table S2\#6-7), and two Tilt Modules (Figure 1\#3-4; Table S4 \#3-4). The platform is enclosed in a Tera Universal 12'x16' HEPA filtered isolation clean room and the STAR has a HEPA filtered hood. Plates and reservoirs are barcoded and scanned with a barcode reader for easy recording, tracking and coordination.

\section{Software}

Workflows were developed and all components of the Hamilton STAR system are operated with the Hamilton Microlab STAR Venus 4 software base package v4.5.0.7977. Scheduling uses the Hamilton Venus Dynamic Scheduler 5.1. Workflow scripts are available from GitHub: https://github.com/AllenInstitute/aics-automated-cell-culture-workflow. Calibration, priming, and initialization information can be found in the Supplement.

\section{Microscopy}

\section{Cell culture morphology and confluency}

The morphology and confluency of the cells were monitored every 4 days with the Celigo Imaging Cytometer (Figure 1 \#19; Table S4 \#19). Bright field images of well overviews were collected at 10X magnification using a broadband white light source. Images of well overviews for 6-well (276 tiles/well) and 96-well plates (1 tile/well; pixel size $=1 \mu \mathrm{m}$ ), were acquired and processed. Quantitative ranking features such as confluency, colony area, colony texture, and number of colonies were measured using llastik pixel classifier (Berg et al., 2019) and CellProfiler (Carpenter et al., 2006; Kamentsky et al., 2011; McQuin et al., 2018) (https://cellprofiler.org/). The Ilastik and CellProfiler pipelines are available from GitHub (https://github.com/AllenInstitute/aicsautomated-cell-culture-workflow). The cytometer was operated with the Celigo Software v4.1.3.0 and the Hamilton driver for Nexcelom Celigo v1.1.

Images of cells in $10 \mathrm{~cm}$ dishes and 6-well plates (Figure 5A) were imaged using a Nikon Ts2 inverted tissue culture microscope with phase contrast equipped with a DS-Fi3 color sCMOS camera and operated with NIS-Element software (Nikon). Images were acquired with the following Nikon CFI60 objective lenses: Plan 2X (N.A. 0.06, W.D. $7.5 \mathrm{~mm}$, F.O.V. $25 \mathrm{~mm}$, PhL), Plan Fluor Phase Contrast DL 4X (N.A. 0.13, W.D. $17.1 \mathrm{~mm}$, F.O.V. $25 \mathrm{~mm}$, PhL), Achromat Phase Contrast ADL 10X (N.A. 0.25, W.D $6.2 \mathrm{~mm}$, F.O.V $22 \mathrm{~mm}$, Ph1) and Achromat Phase Contrast ADL LWD 20X (N.A. 0.4, W.D $3.1 \mathrm{~mm}$, F.O.V 22 mm, Ph1, Fixed Correction $1.2 \mathrm{~mm}$ ).

\section{High resolution imaging of hiPSC}

Imaging of AICS-0011 cell line (TOM20-mEGFP, mitochondria, Figure 6A\&B) was performed on a Zeiss spinning-disk microscope with a 100X/1.2 NA W C-Apochromat Korr UVvis IR objective (Zeiss) and a 1.2X tube lens adapter for a final magnification of 120X, a CSU-x1 spinning-disk head (Yokogawa) and Orca Flash 4.0 camera (Hamamatsu; pixel size $0.108 \mu \mathrm{m}$ in xy after $2 \times 2$ binning and $0.29 \mu \mathrm{m} z$ step size). Standard laser lines (405, 488, 561, $640 \mathrm{~nm}$ ), primary dichroic (RQFT 405, 488, 568, $647 \mathrm{~nm}$ ) and the following Band Pass (BP) filter sets (Chroma) were used for fluorescent imaging: $450 / 50 \mathrm{~nm}$ for detection of NucBlue Live dye (Thermo Fischer Scientific), 525/50 nm for detection of mEGFP, and 706/95 nm for detection of CellMask Deep Red (CMDR) dye (Thermo Fischer Scientific). Cells were imaged in phenol redfree $\mathrm{mTeSR} 1$, within an incubation chamber maintaining $37^{\circ} \mathrm{C}$ and $5 \% \mathrm{CO}_{2}$. Bright field images were acquired using an LED light source with peak emission of $740 \mathrm{~nm}$ with narrow range and a BP filter 706/95 $\mathrm{nm}$ for bright field light collection. 
Automated hiPS cell culture

Allen Institute for Cell Science

\section{Cell and nucleus segmentation and single cell feature extraction}

We applied the deep-learning based cell and nucleus instance segmentation algorithm from the Allen Cell and Structure Segmenter (Chen et al., 2018) to the cell membrane (CMDR dye) and DNA (NucBlue Live dye) channels to obtain single cell and nucleus segmentations (Viana et al., 2020). Features were calculated and extracted from these segmentations. Size and volume were computed using a voxel size of $0.108 \mu m^{3}$ in $x-y-z$ dimension. Sphericity of the nucleus was calculated by mapping the surface area of the object of interest to the surface area of a mathematically perfect sphere with similar volume. The local cell density was estimated by the total number of nuclei in a field of view. The counting of the total number of complete nuclei was performed manually by an expert.

\section{Quality controls}

\section{Cell and colony morphology assessment}

Cell morphology was examined 4 days post-seeding. Wells of 6 -well plates were passaged when $70-85 \%$ confluent and displaying typical morphology associated with stemness and undifferentiated hiPSC cultures (Roberts et al., 2017). Exclusion criteria for passaging included but were not limited to under- or over-confluency, $>1 \%$ differentiating cells, $>5 \%$ cell death, and evidence of contamination. To ensure proper growth of passaging cultures, cell viability and confluency were measured. The viability (\% of viable cells per well) was obtained with a Vi-Cell XR Series Cell Viability Analyzer. Well confluency (\%) was obtained by calculating the fraction of segmented colony area over the total well area. Segmentation output of overview images of each well were created using CellProfiler. 96-well plates imaging QC requirements were as follow: < 3 occurrences of either 1) colonies presenting atypical crater-like morphology, 2) colonies completely lifted (ball-like morphology), 3) colonies with edges lifting and 4) less than $5 \%$ of cells showing signs of differentiation (Figure 5D, E\&G).

\section{Stemness marker expression assessment}

Cells were harvested at $70-85 \%$ confluency, fixed, and frozen at $-80^{\circ} \mathrm{C}$ for later processing in batches. Half of the cell sample was stained for transcription factors with anti-Nanog Alexa Fluor® 647, anti-Oct3/4 Alexa Fluor® 647, and Mouse IgG1 k Isotype Control Alexa Fluor® 647 (all BD Biosciences). The other half of the cell sample was stained for stemness surface markers using anti-SSEA-4 Alexa Fluor 647 and Mouse IgG3 k Isotype Control Alexa Fluor® 647 (all BD Biosciences). Flow cytometry was performed with a CytoFLEX S V4-B2-Y4-R3 Flow Cytometer (Beckman Coulter) equipped with 405, 488, 561, and $637 \mathrm{~nm}$ lasers. Analysis was performed using FlowJo version 10.2 (Treestar). A gating strategy using isotype controls was used to determine the $\%$ of positive cells. The commonly used guideline of $>85 \%$ stemness-marker expression was used to verify stemness of cells (Baghbaderani et al., 2015). Further details for the staining method, gating and analysis can be found in the Supplement, and associated gating histograms in Figure S1.

\section{Cytogenetic stability analysis}

Cells were seeded in GFR Matrigel-coated T-25 Falcon Culture Flasks with Canted Necks (Corning) and cultured for $24 \mathrm{hrs}$. Flasks were then filled with culture media and sent for G-band karyotyping analysis by Diagnostic Cytogenetics, Inc. 20 metaphase cells were counted, 18 metaphase cells analyzed, and two metaphase cells karyotyped per cell sample. Further details for karyotyping reagents can be found in Table S1. 
Automated hiPS cell culture

Allen Institute for Cell Science

\section{Statistical analysis}

Unless indicated, we used two-way analysis of variance (ANOVA) multiparameter analyses through (software) to determine significant differences between manual and automated cell culture methods.

\section{Acknowledgements}

We thank Jenny Burn for editing the video tutorial, Alan R. Horwitz and the Allen Institute for Cell Science Team for helpful discussions. The WTC line that we used to create our geneedited cell lines was provided by the Bruce R. Conklin Laboratory at the Gladstone Institute and UCS. We wish to acknowledge the Allen Institute for Cell Science founder, Paul G. Allen, for his vision, encouragement and support.

\section{Author Contributions}

Conceptualization, S.M.R, W.W., R.N.G., N.G., Methodology, M.E.C., B.W.G., J.A., M.A.F., A.H., M.C.H., I.A.M., A.M.N., D.J.T., C.Y., R.N.G., N.G., Software, M.J.S-B., Validation, M.E.C., B.W.G., J.A., A.B., M.A.F., A.H., M.C.H., W.L., I.A.M., A.M.N., W.J.T., D.J.T., C.Y., N.G., Formal Analysis, M.E.C., A.B., D.J.T., C.Y., Investigation, M.E.C., B.W.G., M.C.H., W.L., W.J.T., Resources, W.W., Data Curation, M.E.C., A.B., M.A.F., A.H., M.C.H., W.L., I.A.M., A.M.N., W.J.T., N.G., Writing - Original Draft, M.E.C., B.W.G., A.B., D.J.T. , C.Y., N.G., Writing - Review \& Editing, M.E.C., B.W.G., A.B., M.A.F., I.A.M., A.M.N., S.M.R., D.J.T., C.Y., R.N.G., N.G., Visualization, M.E.C., B.W.G., A.B., T.P.D., D.J.T., C.Y., N.G., Supervision, S.M.R., W.W., R.N.G., N.G., Project Administration, I.A.M., N.G.

\section{References}

Archibald, P.R.T., Chandra, A., Thomas, D., Chose, O., Massouridès, E., Laâbi, Y., and Williams, D.J. (2016). Comparability of automated human induced pluripotent stem cell culture: a pilot study. Bioprocess Biosyst. Eng. 39, 1847-1858.

Baghbaderani, A.A., Tian, X., Neo, B.H., Burkall, A., Dimezzo, T., Sierra, G., Zeng, X., Warren, K., Kovarcik, D.P., Fellner, T., et al. (2015). cGMP-Manufactured Human Induced Pluripotent Stem Cells Are Available for Pre-clinical and Clinical Applications. Stem Cell Rep. 5, 647-659.

Berg, S., Kutra, D., Kroeger, T., Straehle, C.N., Kausler, B.X., Haubold, C., Schiegg, M., Ales, J., Beier, T., Rudy, M., et al. (2019). ilastik: interactive machine learning for (bio)image analysis. Nat. Methods 16, 1226-1232.

Carpenter, A.E., Jones, T.R., Lamprecht, M.R., Clarke, C., Kang, I.H., Friman, O., Guertin, D.A., Chang, J.H., Lindquist, R.A., Moffat, J., et al. (2006). CellProfiler: image analysis software for identifying and quantifying cell phenotypes. Genome Biol. 7, R100.

Chan, S.W., Rizwan, M., and Yim, E.K.F. (2020). Emerging Methods for Enhancing Pluripotent Stem Cell Expansion. Front. Cell Dev. Biol. 8. 
Automated hiPS cell culture

Allen Institute for Cell Science

Chatterjee, I., Li, F., Kohler, E.E., Rehman, J., Malik, A.B., and Wary, K.K. (2015). Induced Pluripotent Stem (iPS) Cell Culture Methods and Induction of Differentiation into Endothelial Cells. In Induced Pluripotent Stem (IPS) Cells, K. Turksen, and A. Nagy, eds. (New York, NY: Springer New York), pp. 311-327.

Chen, J., Ding, L., Viana, M.P., Hendershott, M.C., Yang, R., Mueller, I.A., and Rafelski, S.M. (2018). The Allen Cell Structure Segmenter: a new open source toolkit for segmenting 3D intracellular structures in fluorescence microscopy images. BioRxiv 491035.

Conway, M.K., Gerger, M.J., Balay, E.E., O'Connell, R., Hanson, S., Daily, N.J., and Wakatsuki, T. (2015). Scalable 96-well Plate Based iPSC Culture and Production Using a Robotic Liquid Handling System. J. Vis. Exp. JoVE.

Crombie, D.E., Daniszewski, M., Liang, H.H., Kulkarni, T., Li, F., Lidgerwood, G.E., Conquest, A., Hernández, D., Hung, S.S., Gill, K.P., et al. (2017). Development of a Modular Automated System for Maintenance and Differentiation of Adherent Human Pluripotent Stem Cells: SLAS Discov. Adv. Life Sci. RD.

Daniszewski, M., Crombie, D.E., Henderson, R., Liang, H.H., Wong, R.C.B., Hewitt, A.W., and Pébay, A. (2018). Automated Cell Culture Systems and Their Applications to Human Pluripotent Stem Cell Studies. SLAS Technol. Transl. Life Sci. Innov. 23, 315-325.

Haenel, F., and Garbow, N. Cell Counting and Confluency Analysis as Quality Controls in CellBased Assays. 5.

Hussain, W., Moens, N., Veraitch, F.S., Hernandez, D., Mason, C., and Lye, G.J. (2013). Reproducible culture and differentiation of mouse embryonic stem cells using an automated microwell platform. Biochem. Eng. J. 77, 246-257.

Jaccard, N., Griffin, L.D., Keser, A., Macown, R.J., Super, A., Veraitch, F.S., and Szita, N. (2014). Automated method for the rapid and precise estimation of adherent cell culture characteristics from phase contrast microscopy images. Biotechnol. Bioeng. 111, 504-517.

Kamentsky, L., Jones, T.R., Fraser, A., Bray, M.-A., Logan, D.J., Madden, K.L., Ljosa, V., Rueden, C., Eliceiri, K.W., and Carpenter, A.E. (2011). Improved structure, function and compatibility for CellProfiler: modular high-throughput image analysis software. Bioinformatics 27, 1179-1180.

Kami, D., Watakabe, K., Yamazaki-Inoue, M., Minami, K., Kitani, T., Itakura, Y., Toyoda, M., Sakurai, T., Umezawa, A., and Gojo, S. (2013). Large-scale cell production of stem cells for clinical application using the automated cell processing machine. BMC Biotechnol. 13, 102.

Koike, H., Kubota, K., Sekine, K., Takebe, T., Ouchi, R., Zheng, Y.-W., Ueno, Y., Tanigawa, N., and Taniguchi, H. (2012). Establishment of automated culture system for murine induced pluripotent stem cells. BMC Biotechnol. 12, 81.

Konagaya, S., Ando, T., Yamauchi, T., Suemori, H., and Iwata, H. (2015). Long-term maintenance of human induced pluripotent stem cells by automated cell culture system. Sci. Rep. 5, 16647.

Kreitzer, F.R., Salomonis, N., Sheehan, A., Huang, M., Park, J.S., Spindler, M.J., Lizarraga, P., Weiss, W.A., So, P.-L., and Conklin, B.R. (2013). A robust method to derive functional neural crest cells from human pluripotent stem cells. Am. J. Stem Cells 2, 119-131. 
Automated hiPS cell culture

Allen Institute for Cell Science

Le, M.N.T., and Hasegawa, K. (2019). Expansion Culture of Human Pluripotent Stem Cells and Production of Cardiomyocytes. Bioengineering 6, 48.

Mahla, R.S. (2016). Stem Cells Applications in Regenerative Medicine and Disease Therapeutics. Int. J. Cell Biol. 2016.

McQuin, C., Goodman, A., Chernyshev, V., Kamentsky, L., Cimini, B.A., Karhohs, K.W., Doan, M., Ding, L., Rafelski, S.M., Thirstrup, D., et al. (2018). CellProfiler 3.0: Next-generation image processing for biology. PLOS Biol. 16, e2005970.

Paull, D., Sevilla, A., Zhou, H., Hahn, A., Kim, H., Napolitano, C., Tsankov, A., Shang, L., Krumholz, K., Jagadeesan, P., et al. (2015). Automated, high-throughput derivation, characterization and differentiation of induced pluripotent stem cells. Nat. Methods 12.

Roberts, B., Haupt, A., Tucker, A., Grancharova, T., Arakaki, J., Fuqua, M.A., Nelson, A., Hookway, C., Ludmann, S.A., Mueller, I.A., et al. (2017). Systematic gene tagging using CRISPR/Cas9 in human stem cells to illuminate cell organization. Mol. Biol. Cell 28, 2854-2874.

Schulz, T.C., Palmarini, G.M., Noggle, S.A., Weiler, D.A., Mitalipova, M.M., and Condie, B.G. (2003). Directed neuronal differentiation of human embryonic stem cells. BMC Neurosci. 4, 27.

Terstegge, S., Laufenberg, I., Pochert, J., Schenk, S., Itskovitz-Eldor, J., Endl, E., and Brüstle, O. (2007). Automated maintenance of embryonic stem cell cultures. Biotechnol. Bioeng. 96, 195201.

Thomas, R.J., Anderson, D., Chandra, A., Smith, N.M., Young, L.E., Williams, D., and Denning, C. (2009). Automated, scalable culture of human embryonic stem cells in feeder-free conditions. Biotechnol. Bioeng. 102, 1636-1644.

Viana, M.P., Chen, J., Knijnenburg, T.A., Vasan, R., Yan, C., Arakaki, J.E., Bailey, M., Berry, B., Borensztejn, A., Brown, J.M., et al. (2020). Robust integrated intracellular organization of the human iPS cell: where, how much, and how variable? BioRxiv 2020.12.08.415562.

Walt, S. van der, Schönberger, J.L., Nunez-Iglesias, J., Boulogne, F., Warner, J.D., Yager, N., Gouillart, E., and Yu, T. (2014). scikit-image: image processing in Python. PeerJ 2, e453.

Wang, Y., Cheng, L., and Gerecht, S. (2014). Efficient and Scalable Expansion of Human Pluripotent Stem Cells Under Clinically Compliant Settings: A View in 2013. Ann. Biomed. Eng. 42, 1357-1372.

Webb, D.J., and Brown, C.M. (2013). Epi-Fluorescence Microscopy. Methods Mol. Biol. Clifton NJ 931, 29-59. 